\title{
Features and Spread Mechanism of Thermal Runaway for Electric Car Batteries
}

\author{
Xiaowei $\mathrm{Li}^{1}$, Fei Zhao ${ }^{1 *}$, Jiangli Hou ${ }^{1}$, Wei Guo ${ }^{2}$ \\ ${ }^{1}$ Automobile Engineering Department, Hebei Vocational University of Technology and Engineering, Xingtai 054000, China \\ ${ }^{2}$ Xingtai Branch of Hebei Institute of Special Equipment Supervision and Inspection, Xingtai 054000, China
}

Corresponding Author Email: zhaofei7517@126.com

https://doi.org/10.18280/ijht.390404

Received: 15 April 2021

Accepted: 1 July 2021

\author{
Keywords: \\ electric cars, batteries, thermal runaway, \\ thermal spread
}

\begin{abstract}
Thermal runaway of the battery pack is a main safety accident of lithium-ion batteries. To improve the safety of lithium-ion batteries, it is of great significance to explore the features and development mechanism of thermal runaway. However, the previous studies mainly focus on a single triggering condition of thermal runaway, and fail to achieve a high modeling accuracy. Hence, this paper probes deep into the features and spread mechanism of thermal runaway for electric car batteries. Firstly, the thermo-physical (TP) parameters were acquired from the batteries, and several key parameters were identified, including the heat transfer parameters in the chamber of accelerating rate calorimeter (ARC), and the thermal parameters of battery debris. Next, the thermal runaway features of the batteries were discussed in stages, and the internal heat yield of batteries was calculated for each stage. After that, the thermal runaway spread was modeled, and a discussion was held on the influence of post-thermal runaway TP parameter changes over the spread features of thermal runaway. The proposed model was proved effective and accurate through experiments.
\end{abstract}

\section{INTRODUCTION}

The electrification of cars has received lots of social attention, as the most promising development direction of automobile power [1-5]. Batteries are the core component of electric cars, which determine the maximum power and maximum mileage of the cars. To promote electric cars, it is critical to improve the service life, charging speed, energy density, and safety of batteries [6-9]. Thermal runaway of the battery pack is a main safety accident of lithium-ion batteries. The potential causes of the accident include quality problems, mechanical damage, and design defects. Once thermal runaway occurs, the passengers will suffer huge property losses and heavy causalities [10-16].

Domestic and foreign scholars have dug deep into the thermal runaway mechanism of batteries, and proposed various ways to suppress the thermal runaway and thermal spread [17-20]. The hybrid thermal management system (HTMS) of lithium-ion batteries involves complex factors, making it difficult to optimize the system design. Zhang et al. [21] designed an HTMS based on phase change materials, liquid cooling, and heat pipes, and established an accurate and reliable numerical model for heat transfer.

To prevent thermal runaway and degradation, the working temperature for the lithium-ion batteries in modern electric cars should be controlled within the allowable range. Afzal et al. [22] numerically simulated the influence of operating parameters (volumetric heat yield, conduction-convection parameter, Reynolds number, and aspect ratio) on the thermal behavior of prismatic batteries, and discussed the effects of Reynolds number and channel spacing on mean pressure and mean Nusselt number. Burd et al. [23] evaluated the lightweight material replacement cost and its time variation for battery electric vehicles (BEVs) made of advanced highstrength steel and aluminum, weighed the costs of car body and closures through process-based cost modeling with a proper mass scaling factor, and computed the costs of batteries, motor, chassis, and other car systems. Wang et al. [24] used 18,650 cylindrical lithium-ion batteries to determine the factors leading to the spread of thermal runaway in the battery pack, and made two discoveries through experiments: the cyclic time has little impact on thermal propagation, and the thermal runaway is more likely to spread when the temperature of the adjacent battery increases faster than $0.36^{\circ} \mathrm{C} / \mathrm{s}$. Based on state representation method, Jiang et al. [25] proposed a datadriven approach for fault diagnosis and thermal runaway warming of lithium-ion batteries: the real-time state of each battery is used to characterize the internal features of that battery, and the state change is recorded to realize the diagnosis of battery faults. Li et al. [26] constructed a thermal runaway model through internal short-circuit simulation, and solved the model on Ansys Fluent, thereby realizing the mass and heat transfers between batteries and heat pipes.

The previous studies mainly focus on the modeling of thermal runaway for different batteries, and the thermal runaway features of batteries under a single triggering condition. However, thermal runaway can be triggered by various conditions. Most studies fail to recognize the thermophysical (TP) parameters of battery materials after thermal runaway. To solve the problem, this paper firstly identifies the TP parameters of post-thermal runaway batteries, and applies the results to analyze the features and spread mechanism of thermal runaway for electric car batteries. Section 2 acquires the TP parameters from the batteries, and recognizes several key parameters, including the heat transfer parameters in the chamber of accelerating rate calorimeter (ARC), and the 
thermal parameters of battery debris. Section 3 discusses the thermal runaway features of the batteries in stages, and calculates the internal heat yield of batteries for each stage. Section 4 models the spread of thermal runaway, and discusses the influence of post-thermal runaway TP parameter changes over the spread features of thermal runaway. The proposed model was proved effective and accurate through experiments.

\section{RECOGNITION OF BATTERY TP PARAMETERS AFTER THERMAL RUNAWAY}

To estimate the TP parameters of the batteries after being internally damaged by thermal runaway, the aluminum shell battery debris, which obviously bulged in the ARC thermal runaway experiment, was tested. Figure 1 shows the flow of TP parameter acquisition and model analysis.

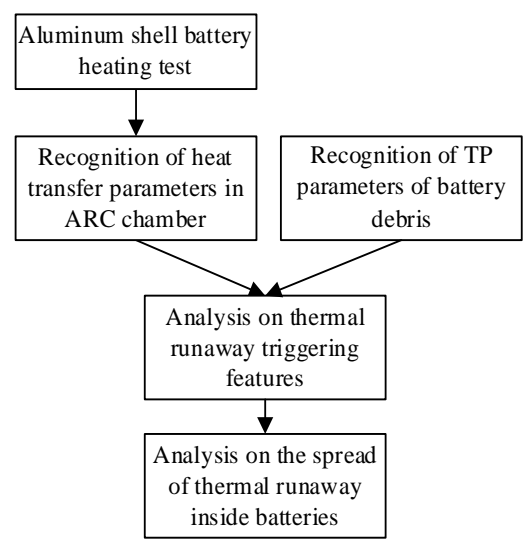

Figure 1. Flow of TP parameter acquisition and model analysis

On COMSOL Multiphysics software, a three-dimensional (3D) model was established for aluminum shell heating. The ambient temperature of the aluminum shell was set as the mean wall temperature of ARC $\psi_{A R C}$. The aluminum shell exchanges heat with the environment via both convection and radiation. Let $\gamma$ be the radiation coefficient; $\mu_{A l}$ be the normal conduction coefficient of the boundary material of aluminum shell; $B O$ be the Boltzmann constant; $\psi$ be the temperature of aluminum shell. Then, we have:

$$
-\mu_{A l} \frac{\partial \psi}{\partial \vec{n}}=f\left(\psi-\psi_{A R C}\right)+\gamma B O\left(\psi^{*}-\psi_{A R C}^{*}\right)
$$

The mean temperature and temperature difference between batteries and the environment determine the size of the convection coefficient between the two. The temperature difference between the aluminum shell and the environment was divided into six segments with an interval of $10^{\circ} \mathrm{C}$. The six corresponding convection coefficients are denoted as $f_{1}$ $f_{6}$. Then, the comprehensive expression of the convection coefficients can be expressed as:

$$
f= \begin{cases}f_{1} & \left(\psi-\psi_{A R C}\right) \leq 10^{\circ} \mathrm{C} \\ f_{2} & 10^{\circ} \mathrm{C}<\left(\psi-\psi_{A R C}\right) \leq 20^{\circ} \mathrm{C} \\ f_{3} & 20^{\circ} \mathrm{C}<\left(\psi-\psi_{A R C}\right) \leq 30^{\circ} \mathrm{C} \\ f_{4} & 30^{\circ} \mathrm{C}<\left(\psi-\psi_{A R C}\right) \leq 40^{\circ} \mathrm{C} \\ f_{5} & 40^{\circ} \mathrm{C}<\left(\psi-\psi_{A R C}\right) \leq 50^{\circ} \mathrm{C} \\ f_{6} & 50^{\circ} \mathrm{C}<\left(\psi-\psi_{A R C}\right) \leq 60^{\circ} \mathrm{C}\end{cases}
$$

where, $f_{1}-f_{6}$ and $\gamma$ are the seven parameters to be recognized during the heat exchange in ARC chamber.

Table 1. Target heat transfer parameters in ARC chamber

\begin{tabular}{cccccccc}
\hline $\begin{array}{c}\text { Temperature } \\
\text { difference }\end{array}$ & - & $0 \sim 10$ & $10 \sim 20$ & $20 \sim 30$ & $30 \sim 40$ & $40 \sim 50$ & $50 \sim 60$ \\
\hline $\begin{array}{c}\text { Parameter } \\
\text { Optimal }\end{array}$ & $\gamma$ & $f_{1}$ & $f_{2}$ & $f_{3}$ & $f_{4}$ & $f_{5}$ & $f_{6}$ \\
$\begin{array}{c}\text { initial value } \\
\text { Optimal }\end{array}$ & 0.36 & 2.6 & 3.6 & 4.6 & 5.6 & 6.6 & 7.6 \\
$\begin{array}{c}\text { range } \\
\text { Optimal }\end{array}$ & $0.01 \sim 08$ & $0.01 \sim 250.01 \sim 250.01 \sim 250.01 \sim 250.01 \sim 250.01 \sim 25$ \\
result & 0.38 & 2.35 & 4.03 & 4.56 & 5.38 & 5.79 & 6.81 \\
\hline
\end{tabular}

Table 2. Target thermal parameters of the debris

\begin{tabular}{ccccc}
\hline Parameter & $\mu_{c 1} \quad \mu_{c 2}$ & $\begin{array}{c}\text { Specific } \\
\text { heat } \\
\text { capacity }\end{array}$ & $\begin{array}{c}\text { Reciprocal of the thermal } \\
\text { resistance between aluminum } \\
\text { shell and internal material }\end{array}$ \\
\hline $\begin{array}{c}\text { Optimal } \\
\text { initial value }\end{array}$ & 0.5 & 15 & 1,000 & 110 \\
$\begin{array}{c}\text { Optimal } \\
\text { range }\end{array}$ & $0 \sim 10 \sim 30$ & $0 \sim 1,600$ & $0 \sim 1,100$ \\
$\begin{array}{c}\text { Optimal } \\
\text { result }\end{array}$ & 0.368 .19 & 756.34 & 35.72 \\
\hline
\end{tabular}

Table 1 shows the recognition results of the seven optimal parameters. Obviously, the convection coefficients of the aluminum shell in ARC chamber increases with the temperature difference between the aluminum shell and the environment. Based on the final recognition results, the calculated surface temperature of the aluminum shell was compared with the temperature measured through experiments The calculated results on the seven parameters were imported to the ARC heating model for battery debris.

After the thermal runaway test, a battery debris heating test was carried out, in which the ARC parameters were configured the same as those for aluminum shell heating test. The batteries suffering from thermal runaway would not deform greatly, due to the pre-tensioning of the end plates. The variation in its conduction coefficients and heat capacity mainly comes from combustion and eruption. Therefore, this paper identifies the heat capacity and conduction coefficients of undeformed batteries after thermal runaway as the target TP parameters. Let $\xi_{1}$ and $\xi_{2}$ be the material thickness and air thickness in the target battery debris, respectively. Then, the ARC test debris can be viewed as the superposition of uniform layers of the material and the air.

Let $\mu_{c}, \mu_{c 1}$, and $\mu_{c 2}$ be the conduction coefficients of the batteries, the debris, and the air perpendicular to c, respectively; $\mu_{b}, \mu_{b 1}$, and $\mu_{b 2}$ be the conduction coefficients of the batteries, the debris, and the air parallel to $b$, respectively; for the air, $\mu_{c 2}=\mu_{b 2}=\mu_{A}$. The conduction coefficient of the batteries perpendicular to pole piece $c$ can be calculated by:

$$
\mu_{c}=\frac{\xi_{1}+\xi_{2}}{\frac{\xi_{1}}{\mu_{c 1}}+\frac{\xi_{2}}{\mu_{c 2}}}
$$

The conduction coefficient of the batteries parallel to pole piece $b$ can be calculated by:

$$
\mu_{b}=\frac{\mu_{b 1} \xi_{1}+\mu_{b 2} \xi_{2}}{\xi_{1}+\xi_{2}}
$$


Table 2 lists the recognition results on the TH parameters of the debris, including $\mu_{c 1}$, specific heat capacity, and the reciprocal of the thermal resistance between aluminum shell and internal material.

\section{THERMAL RUNAWAY FEATURES OF BATTERIES}

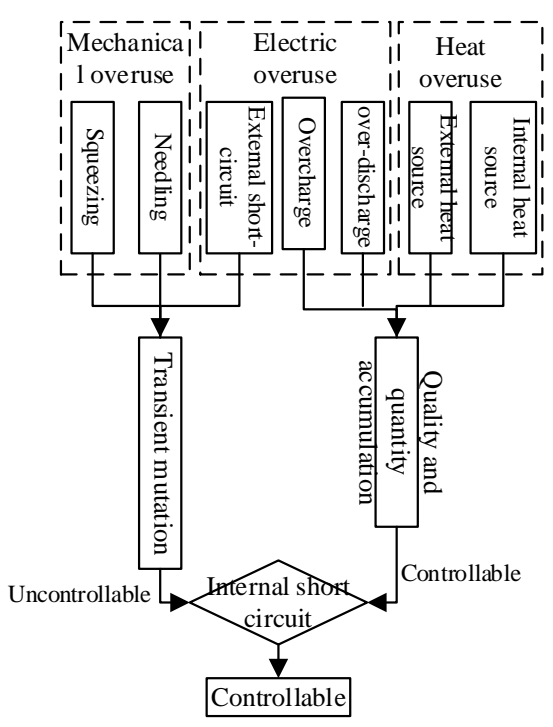

Figure 2. Flow of generation of battery thermal runaway

The thermal runaway of batteries could be triggered by three types of factors: mechanical overuse, electric overuse, and heat overuse. The three kinds of factors have certain correlations and differences. Figure 2 illustrates the flow of the generation of battery thermal runaway. The thermal runaway of batteries is often accompanied by internal short circuit, which arises from the transient mutation caused by the external short circuit, a product of mechanical and electric overuses, and from the quality and quantity accumulation caused by the overcharge and over-discharge, the results of heat and electric overuses. Once short circuit occurs inside the batteries, thermal runaway will quickly ensue.

There are complex chemical reactions inside batteries. Let $v_{T}$ be the total heat yield rate of secondary reactions in lithiumion batteries; $v_{S E I}$ be the heat yield rate of solid electrolyte interface decomposition; $v_{F}$ and $v_{Z}$ be the heat yield rates of negative and positive pole materials reacting with electrolyte, respectively; $v_{D}$ be the heat yield rate of electrolyte decomposition. The units of $v_{T}, v_{S E I}, v_{F}, v_{Z}$, and $v_{D}$ are all $\mathrm{W} / \mathrm{m}^{3}$. Without considering the reactions within the batteries that generate a limited amount of heat, $v_{T}$ can be expressed as:

$$
v_{T}=v_{S E I}+v_{F}+v_{Z}+v_{D}
$$

Let $\varphi$ be the rate constant of electrochemical reactions; $\psi_{B}$ be the temperature of lithium-ion batteries; $G$ be the preexponential factor of electrochemical reactions; $u$ be the concentration of the substances participating in electrochemical reactions; $S$ be the radius of cylindrical batteries; $Q_{g}$ be the activation energy of electrochemical reactions. Then, the heat yield of the electrochemical reactions of the lithium-ion batteries can be calculated by:

$$
\phi=G e^{-\frac{Q_{g}}{S \psi}}
$$

$$
\phi=\frac{d u}{d t}
$$

Based on the reactive substance contents and activation energy of secondary electrochemical reactions inside the batteries, the heat yield and heat yield rate of thermal runaway can be estimated and analyzed based on reaction kinetics. According to the sequence of secondary electrochemical reactions, the analysis could be divided into four stages: Stage $\mathrm{I}$, heat generation through decomposition of solid electrolyte interface; Stage II, heat generation through the reaction between negative pole material and electrolyte; Stage III, heat generation through the reaction between positive pole material and electrolyte; Stage IV, heat generation through electrolyte decomposition

(1) Stage I

When the battery temperature reaches $70^{\circ} \mathrm{C}-90^{\circ} \mathrm{C}$, the solid electrolyte interface begins to decompose. Let $P R_{S E I}$ be the heat yield rate of decomposition; $C O_{T}$ be the carbon content of negative pole material; $D E_{S E I}$ be the decomposition rate of the solid electrolyte interface. Then, we have:

$$
v_{S E I}=P R_{S E I} \cdot C O_{T} \cdot D E_{S E I}
$$

Let $G_{S E I}$ be the pre-exponential factor of solid electrolyte interface decomposition; $Q_{g-S E I}$ be the activation energy of decomposition; $n_{S E I}$ be the order of decomposition; $u_{S E I}$ be the proportion of active lithium in the interface; $S$ be the gas reaction constant. Then, $D E_{S E I}$ can be calculated by:

$$
\begin{gathered}
D E_{S E I}=G_{S E I} e^{-\frac{Q_{g}-S E I}{S \psi}} u_{S E I}^{n_{S E I}} \\
\frac{d u_{S E I}}{d t}=-D E_{S E I}
\end{gathered}
$$

\section{(2) Stage II}

Due to the decomposition of solid electrolyte interface, the battery temperature continues to rise. When the temperature rises to around $120^{\circ} \mathrm{C}$, the negative pole material will react with the electrolyte. Let $P R_{F}, D E_{F}$, and $C O_{F}$ be the heat yield rate, reaction rate, and pre-exponential factor of negative pole material, respectively. Then, we have:

$$
v_{F}=P R_{F} \cdot C O_{F} \cdot D E_{F}
$$

Let $Q_{g-F}$ be the activation energy of the reaction of negative pole material; $n_{g-F}$ be the order of the reaction; $u_{F}$ be the proportion of the lithium ions in negative pole material that can react with electrolyte; $h_{S E I}$ be the ratio of solid electrolyte interface thickness to the size of active particles. Then, $D E_{F}$ can be calculated by:

$$
\begin{gathered}
D E_{F}=G_{F} e^{-\frac{h_{S E I}}{h_{S E I 0}}} u_{F}^{h_{F-m}} e^{-\frac{Q_{g-F}}{S \psi}} \\
\frac{d h_{S E I}}{d t}=D E_{F}
\end{gathered}
$$

$$
\frac{d u_{F}}{d t}=-D E_{F}
$$




\section{(3) Stage III}

When the battery temperature rises to $180 \sim 200^{\circ} \mathrm{C}$, the positive pole material will react with the electrolyte. Let $P R_{Z}$, $D E_{Z}$, and $C O_{Z}$ be the heat yield rate, reaction rate, and preexponential factor of positive pole material, respectively; $D E_{Z}$ be the reaction rate of positive pole material. Then, we have:

$$
v_{Z}=P R_{Z} \cdot C O_{Z} \cdot D E_{Z}
$$

Let $G_{Z}$ be the pre-exponential factor of the reaction of positive pole material; $Q_{g-Z}$ be the activation energy of the reaction; $n_{Z-o 1}$ and $n_{Z-o 2}$ be the orders of the reaction; $g$ be the percentage of positive material participating in reaction. Then, $D E_{Z}$ can be calculated by:

$$
\begin{gathered}
D E_{Z}=G_{Z} g^{n_{--01}}(1-g)^{n_{Z-02}} e^{-\frac{Q_{g-2}}{S \psi}} \\
\frac{d g}{d t}=D E_{Z}
\end{gathered}
$$

\section{(4) Stage IV}

When the battery temperature rises to $200 \sim 250^{\circ} \mathrm{C}$, the lithium salt reacts with the solvent of the electrolyte. Let $P R_{D}$ be the heat yield rate of electrolyte decomposition; $C O_{D}$ be electrolyte content; $D E_{D}$ be the reaction rate of the electrolyte. Then, we have:

$$
v_{D}=P R_{D} \cdot C O_{D} \cdot D E_{D}
$$

Let $G_{D}$ be the pre-exponential factor of electrolyte reaction; $Q_{g-D}$ be the activation energy of the reaction; $u_{D}$ be the percentage of the part of the electrolyte not involved in the reaction; $n_{D}$ be the order of the reaction. Then, $D E_{D}$ can be calculated by:

$$
\begin{gathered}
D E_{D}=G_{D} e^{-\frac{Q_{g-D}}{S \psi}} u_{D}^{n_{D}} \\
\frac{d u_{D}}{d t}=-D E_{D}
\end{gathered}
$$

Based on the triggering temperatures of secondary electrochemical reactions, the overall temperature of the overused batteries will continue to increase. When the temperature reaches around $80^{\circ} \mathrm{C}$ (the starting point of solid electrolyte interface decomposition), the batteries enter Stage I and begin to generate heat, and the heat yield of the decomposition is enough to drive up the battery temperature at a constant rate. When the temperature reaches around $120^{\circ} \mathrm{C}$ (the starting point of the reaction between negative pole material and electrolyte), the batteries enter Stage II and generate heat more quickly, under the new secondary reactions. Eventually, the temperature climbs up to $250^{\circ} \mathrm{C}$ (the starting point for stable thermal decomposition of positive pole material). Then, the batteries enter Stage III, and the positive pole material decomposes, releasing active oxygen that can burn with the combustibles in the batteries. In this stage, the central temperature of the batteries will surpass $1,000^{\circ} \mathrm{C}$. Under the high temperature, all secondary electrochemical reactions will be more violent, releasing even more heat. As a result, the temperature will rise again. The mutual promotion process makes the thermal runaway of batteries difficult to suppress and prevent.

\section{MODELING OF THERMAL RUNAWAY SPREAD}

Thermal runaway causes significant changes to the $\mathrm{TH}$ parameters of batteries, which in turn bring changes to the conduction properties between batteries. The conduction changes manifest as the spread of thermal runaway across batteries. Based on the heat yield of thermal runaway, and the $\mathrm{TH}$ parameters obtained in the preceding section, this paper models the spread of thermal runaway of batteries, and discusses how the changing TH parameters of batteries affect the spread of thermal runaway.

The proposed model of thermal runaway spread can simulate the spread of local thermal runaway, which obeys the solid heat transfer law, within batteries. The energy release of thermal runaway can be described by the temperature-based solid heat transfer equation. Considering the anisotropic conduction of batteries, the internal heat source of batteries could be calculated based on the results of ARC adiabatic thermal runaway test, and the exchange boundary conditions between battery temperature and environment temperature were determined, laying the basis for temperature field prediction after thermal runaway.

Let $\varepsilon$ be the core material density of batteries; $S H_{M}$ and $\alpha$ be the specific heat capacity and conduction coefficient of core material, respectively; $a, b$ and $c$ be the subscripts of different directions; $P_{M}$ be heat yield power per unit volume. Then, the heat transfer of battery thermal runaway can be described by:

$$
\varepsilon S H_{M} \frac{d \psi}{d t}=P_{M}+\frac{\partial}{\partial a}\left(\alpha_{a} \frac{\partial \psi}{\partial a}\right)+\frac{\partial}{\partial b}\left(\alpha_{b} \frac{\partial \psi}{\partial b}\right)+\frac{\partial}{\partial c}\left(\alpha_{c} \frac{\partial \psi}{\partial c}\right)
$$

During the thermal runaway of a single battery, the temperature of core material will increase violently till the peak level after reaching $\psi^{\prime}$. Let $\omega_{j}$ be the j-th TH parameter of core material within batteries; $\omega_{j-0}$, and $\Delta \omega_{j}$ be the initial values and variations of the conduction coefficients, density, and specific heat conductivity, respectively; $v$ be the percentage of the heat released by the secondary reactions of

\begin{tabular}{|c|c|c|c|c|c|}
\hline Component & $\begin{array}{c}\text { Aluminum } \\
\text { shell }\end{array}$ & $\begin{array}{c}\text { Negative } \\
\text { pole }\end{array}$ & $\begin{array}{c}\text { Positive } \\
\text { pole }\end{array}$ & Needle & $\begin{array}{c}\text { Film } \\
\text { between } \\
\text { core and } \\
\text { shell } \\
\end{array}$ \\
\hline Material & Aluminum & Copper & Aluminum & Steel & Air \\
\hline Density & 2,800 & 8,980 & 2,800 & 7,950 & $\varepsilon_{A}$ \\
\hline $\begin{array}{c}\text { Specific heat } \\
\text { capacity }\end{array}$ & 910 & 395 & 890 & 465 & $\mathrm{SH}_{A}$ \\
\hline $\begin{array}{c}\text { Conduction } \\
\text { coefficient }\end{array}$ & 239 & 410 & 239 & 45.6 & $100 * \alpha_{A}$ \\
\hline
\end{tabular}
core material. Suppose the thermal parameters of core material increase linearly as the temperature rises from $\psi^{\prime}$ to $\psi^{\prime \prime}$. The entire variation process can be described by:

$$
\omega_{j}(\psi)=\left\{\begin{array}{lc}
\omega_{j, 0} & \psi \leq \psi^{\prime}, v>0 \\
\omega_{j, 0}-\frac{\psi-\psi^{\prime}}{\psi^{\prime \prime}-\psi^{\prime}} \Delta \omega_{j} & \psi>\psi^{\prime}, v>0 \\
\omega_{j, 0}-\Delta \omega_{j} & v=0
\end{array}\right.
$$

Table 3. Battery components and their TH parameters 
Table 3 lists the battery components and their $\mathrm{TH}$ parameters. Note that there is a thin layer of gap between the core and the shell. This paper sets the gap into the air. Considering the different flows and heat transfer states between the static air and the actual air, the conduction coefficient of the thin layer was corrected into 100 times the conduction coefficient $\alpha_{A}$ of the air.

Let $d_{M-C H}$ be the heat yield of the secondary reactions of the core; $d_{M-N A}$ be the heat yield of the short circuit induced by needling. These are two main heat sources of the model. Let $E_{C H}$ and $E_{N A}$ be the total energy released by the two sources, respectively; $U_{C H}$ and $U_{N A}$ be the volumes of the core and needle, respectively. Then, the relationship between the power of the core as a heat source per unit volume and the total heat release can be described by:

$$
E_{C H}=\iint_{\tau} \int_{U_{B A}} d_{M, C H} d U_{C H} d t
$$

The relationship between the power of the needle as a heat source per unit volume and the total heat release can be described by:

$$
E_{N A}=\iint_{\tau} \int_{U_{B A}} d_{M, N A} d U_{N A} d \tau
$$

The total energy $E_{T}$ released by battery thermal runaway roughly equals the sum of $E_{C H}$ and $E_{N A}$. Let $\beta$ be the proportion of $E_{N A}$. Then, we have:

$$
\begin{gathered}
E_{T}=E_{C H}+E_{N A} \\
E_{N A}=\beta \cdot E_{T}
\end{gathered}
$$

Based on the data of adiabatic thermal runaway test on batteries, the total heat yield $E_{T}$ can be calculated as follows: Let $\psi_{0}$ be the initial temperature of the heat yield process; $\varepsilon_{C H}(\psi)$ be core material density; $S_{M-C H}(\psi)$ be the specific heat capacity of core material. Then, we have:

$$
\begin{aligned}
& E_{T}=\int_{U_{C H}} \int_{\psi_{0}}^{\psi^{\prime \prime}} \varepsilon_{C H}(\psi) S H_{M-C E}(\psi) \cdot W_{A R C} d \psi d U_{C H} \\
& W_{A R C}= \begin{cases}0 & \text { Heating or waiting mode } \\
1 & \text { Other modes }\end{cases}
\end{aligned}
$$

The energy released by short circuit at the needling point was subjected to thermocouple simulation of short circuit. Let $\delta(h)$ be the factor function of short circuit power release rate. The energy release rate of the internal short circuit induced by needling can be calculated by:

$$
d_{M-S C}=\frac{1}{U_{S C}} \cdot E_{S C} \cdot \delta(t)
$$

The chemical energy release rate of batteries was defined according to the temperature rise data of ARC adiabatic thermal runaway. The energy release rate of battery thermal runaway is a function of temperature. It is assumed that the heat release rate of adiabatic thermal runaway equals that induced by needling. Let $\psi^{\prime}$ be the triggering temperature of thermal runaway. When the internal temperature of batteries falls below $\psi^{\prime}$, the energy release rate can be calculated based on the temperature rise rate of adiabatic temperature rise rate. When the internal temperature of batteries surpasses $\psi^{\prime}$, the secondary thermochemical reactions become increasingly intense under high temperature. Then, the residual energy will be released at a constant rate, and can be calculated by:

$$
d_{M-C H}=\frac{1}{U_{C H}} \cdot E_{C H} \cdot \frac{d e}{d t}
$$

The heat yield of batteries is so small as to be negligible, when the temperature is below $145^{\circ} \mathrm{C}$. Let $(d \psi / d t)_{A R C}$ be the temperature rise rate of batteries obtained through ARC test. Then, the change rate of $e$ can be expressed as:

$$
\frac{d e}{d t}=\left\{\begin{array}{lc}
\frac{\varepsilon_{C H}(\psi) U_{C H} S H_{M-C E}(\psi)}{U_{C H}}\left(\frac{d \psi}{d t}\right)_{A R C} & \left(0 \leq e \leq 1, \psi_{C H}<\psi \leq \psi^{\prime}\right) \\
S H_{C H} & \left(0 \leq e \leq 1, \psi>\psi_{2}\right) \\
0 & \text { Other conditions }
\end{array}\right.
$$

The insulating layer between the core and the shell is easily affected by the contact thermal resistance, and has a small conduction coefficient. Therefore, the heat transfer effect on the normal direction of the thin layer cannot be ignored. Our model defines this thin layer between the core and the aluminum shell. Let $\alpha_{m-q}$ and $\alpha_{m-h}$ be the normal conduction coefficients of the materials before and behind the thin layer, respectively; $\psi_{q}$ and $\psi_{h}$ be the temperatures of the two materials on the contact surface, respectively; $\alpha_{b}$ be the conduction coefficient of the thin layer, which can be singlelayered or multi-layered; $\xi_{b}$ be the thickness of the thin layer. Then, the heat flow density of the core and the shell can be calculated by:

$$
\begin{aligned}
& -\alpha_{m-q} \frac{\partial \psi_{q}}{\partial \vec{n}}=\frac{\alpha_{b}\left(\psi_{q}-\psi_{h}\right)}{\xi_{b}} \\
& -\alpha_{m-q} \frac{\partial \psi_{q}}{\partial \vec{n}}=\frac{\alpha_{b}\left(\psi_{q}-\psi_{h}\right)}{\xi_{b}}
\end{aligned}
$$

Let $\eta, \theta$, and $\sigma$ be normal convection coefficient, convection coefficient, and radiation coefficient of boundary materials, respectively; $\psi_{A}$ be ambient temperature. The battery surface directly exposed to the environment undergoes heat exchange through convection and radiation. The corresponding boundary equation can be expressed as:

$$
-\eta_{m} \frac{\partial \psi}{\partial \vec{n}}=\theta\left(\psi-\psi_{A}\right)+\sigma \cdot B O\left(\psi^{*}-\Phi_{A}^{*}\right)
$$

The other boundary conditions of our model were configured, and the exchange parameters between batteries and environment were adjusted, according to the actual results of simulation. Table 4 lists the thermal resistance layers and transfer coefficients. The layers include the first insulating layer, second insulating layer, and the air layer at four places: top surface of the core 1 , side surface of the core 2, bottom of the core 3 , and inside of the upper cover 4 . 
Table 4. Thermal resistance layers and transfer coefficients of thermal runaway induced by needling

\begin{tabular}{|c|c|c|c|c|c|c|c|}
\hline Location & & 1 & & 2 & & 3 & 4 \\
\hline Name & Layer & 1Layer 2 & Layer 1 & 1Layer & Layer & 1 Layer 2 & $\overline{\text { Air layer }}$ \\
\hline$\xi$ & 0.2 & 0.03 & 0.16 & 0.4 & 1.6 & 3 & 2 \\
\hline$\eta$ & 0.23 & 0.05 & 0.23 & 0.05 & 0.23 & 0.05 & 0.05 \\
\hline$\varepsilon$ & 920 & 1.4 & 920 & 1.4 & 920 & 1.4 & 1.4 \\
\hline SH & 1,895 & 1,100 & 1,895 & 1,100 & 1,895 & 1,100 & 1,100 \\
\hline
\end{tabular}

\section{EXPERIMENTS AND RESULTS ANALYSIS}

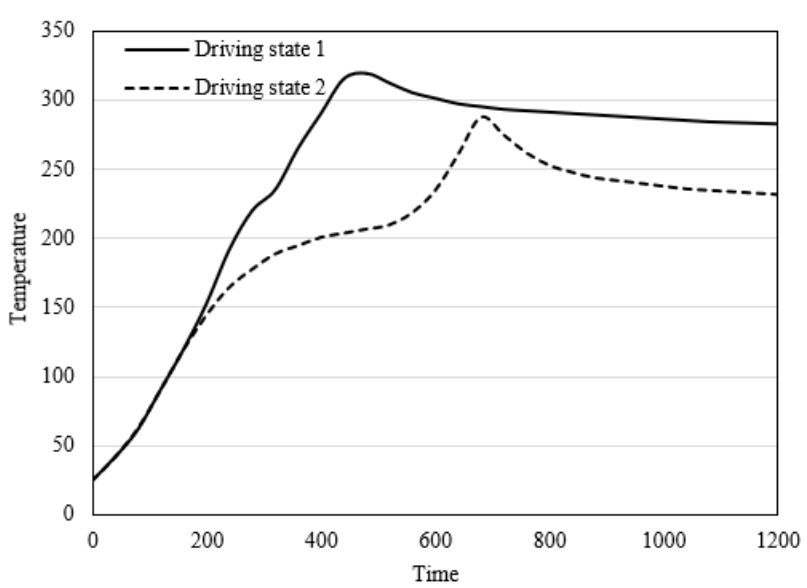

Figure 3. Temperature change at battery detection point under different thermal source temperatures

To capture the changes of thermal runaway features with thermal source temperatures, the temperatures of battery detection point under driving states 1 and 2 of an electric car were compared. Without changing thermal source locations and coefficients of heat release, the temperature of external heat source was initialized as $20^{\circ} \mathrm{C}$. After that, the temperature was increased at $1{ }^{\circ} \mathrm{C}$ per second until the preset level, and then kept stable. Figure 3 intuitively displays the temperature change at battery detection point, i.e., the center of negative pole material, under two driving states.

As shown in Figure 3, the temperature curve of state 1 was generally ahead of that of state 2 , but the peak temperature of state 1 was higher than that of state 2 . Under state 1 , the temperature reached the peak of $324.08^{\circ} \mathrm{C}$ at $512 \mathrm{~s}$; Under state 2 , the temperature peaked at $284.12^{\circ} \mathrm{C}$ at $689 \mathrm{~s}$.

The battery temperature curve under state 1 inflected clearly at $310 \mathrm{~s}$. The temperature rise slowed down, mainly because the batteries mainly rely on secondary electrochemical reactions to increase temperature, after reaching the temperature of external heat source.

The battery temperature curve also inflected clearly at $573 \mathrm{~s}$. The higher the temperature of external heat source, the faster the battery enters thermal runaway, and the higher the peak temperature. After reaching the peak, the battery temperature slowly decreased. The decreasing rate was relatively small, when the external heat source was hot.

To capture the changes of thermal runaway features with coefficients of heat release, the temperatures of battery detection point under charging states 1 and 2 of an electric car were compared. Without changing thermal source locations and heat source temperatures, different coefficients of heat release were configured for batteries. Figure 4 compares the temperature change at battery detection point, i.e., the center of negative pole material, under two charging states.

As shown in Figure 4, the temperature curve under state 2 was gentler than that under state 1 . No peak was observed. Besides, the temperature did not mutate as that under state 1 . The reason is that the coefficient of heat release is relatively large for battery management under state 2 . Hence, the heat can be transmitted to the outside of the aluminum shell quickly to avoid the peak temperature. As a result, it is more difficult for batteries to enter the stage of thermal runaway.

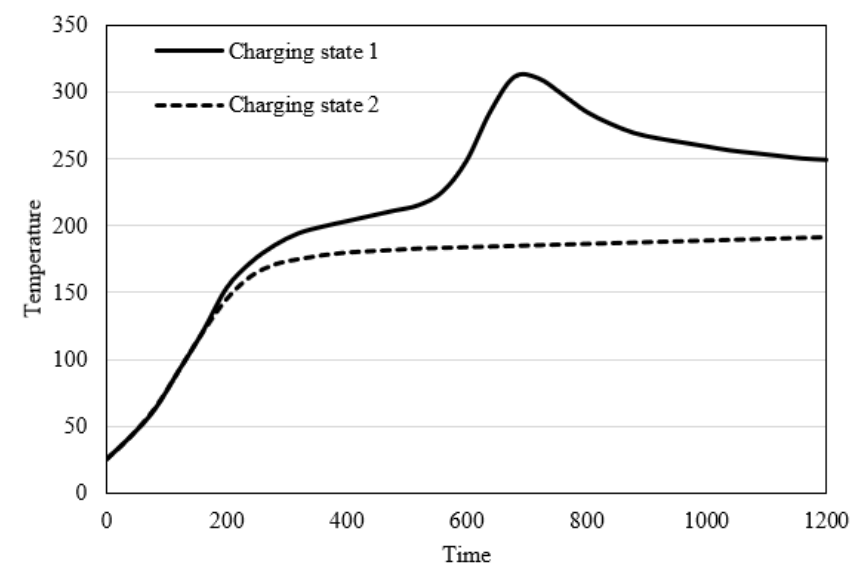

Figure 4. Temperature change at battery detection point under different coefficients of heat release

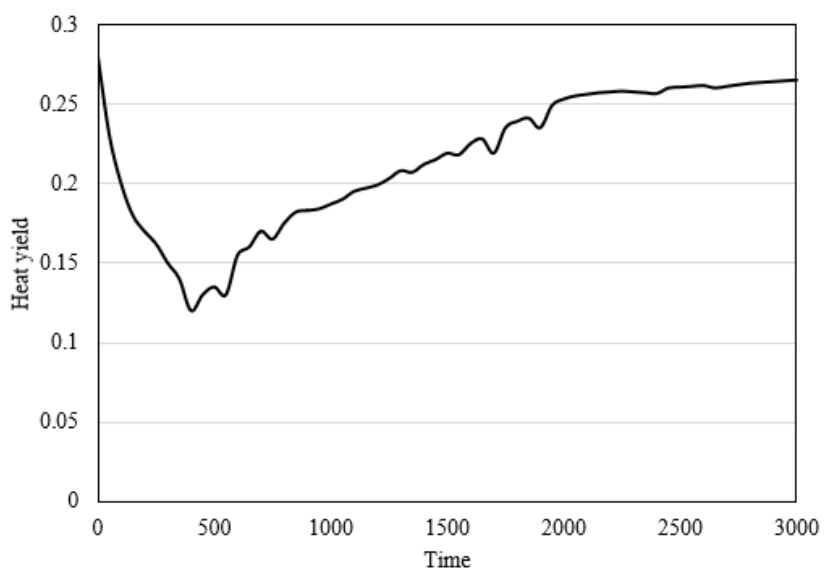

(a) Charging state 1

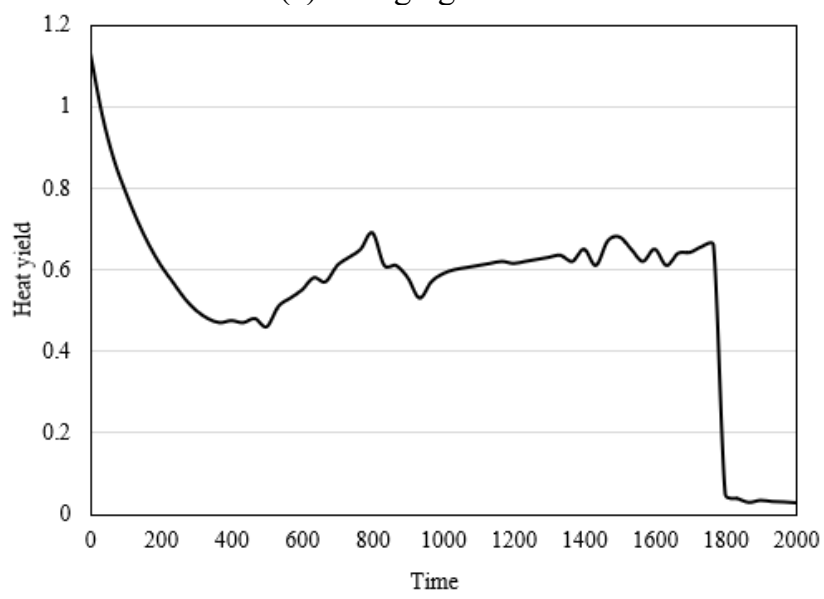

(b) Charging state 2

Figure 5. Total heat yield variation with charging speeds 
To analyze the changes of thermal runaway features with charging speeds, the temperatures of battery detection point under charging states 1 and 2 of an electric car were compared. It can be inferred that the battery temperature curves followed almost the same trend under different charging speeds: the peak temperatures all appeared at around 260 s, reaching about $350^{\circ} \mathrm{C}$.

Figure 5 shows the variation of total heat yield of the batteries' thermal runaway at different charging speeds. Comparing subgraphs (a) and (b), it can be learned that the greater the internal heat yield of the batteries, the more serious the thermal runaway. In subgraph (a) with a relatively low charging speed, the total heat yield of the batteries' thermal runaway plunged quickly from the initial value of $0.27 \mathrm{~W}$ to $0.13 \mathrm{~W}$, and then gradually rose to $0.277 \mathrm{~W}$. In subgraph (b) with a relatively fast charging speed, the total heat yield nosedived quickly from the initial value of $1.12 \mathrm{~W}$ to $0.47 \mathrm{~W}$, and then fluctuated at around $0.6 \mathrm{~W}$.

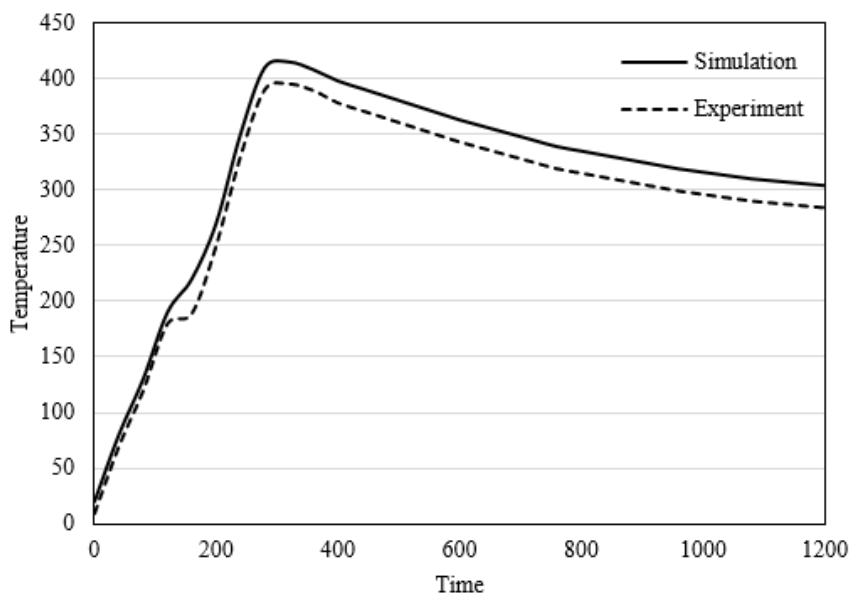

Figure 6. Comparison between experimental results and simulation results

Several experiments were carried out to verify the accuracy of our model for thermal runaway and spread of batteries. During different groups of experiments, the data were collected from temperature sensors at different points. To enhance the reliability, the mean data of all groups were taken as the final results. Figure 6 compares the mean experimental results with the mean simulation results. It can be seen that both sets of data first increased and then declined. The initial temperature of experimental results was lower than that of simulation results, because the lab temperature is $5^{\circ} \mathrm{C}$ lower than the ambient temperature for simulation. The different between the mean experimental results and the mean simulation results was within $5 \%$. Therefore, the proposed model for thermal runaway and spread of batteries is accurate enough for simulation analysis.

Table 5. Fitted convection coefficients of battery pack

\begin{tabular}{ccccccc}
\hline $\begin{array}{c}\text { Mean temperature } \\
\text { Temperature } \\
\text { difference }\end{array}$ & 125 & 116 & 110 & 105 & 98 & 92 \\
$\begin{array}{c}\text { Convection } \\
\text { coefficient }\end{array}$ & 2.37 & 4.05 & 4.63 & 5.34 & 5.79 & 6.81 \\
\hline
\end{tabular}

The proposed model was adopted to compute the thermal runaway spread of battery pack. Table 5 lists the fitted convection coefficients of battery pack. Without considering the slow heat release within batteries, the post-thermal runaway temperature drop rate was calculated, and compared with the experimental result. Through the comparison, the heat yield and slowdown of temperature drop rate were obtained for the batteries after thermal runaway.

Figure 7 presents the curves obtained from internal temperature experiments on batteries. Note that $\psi_{i}$ is the central temperature of the i-th battery starting from the side of the ARC. Table 6 lists the experimental results and simulation results on thermal runaway spread time, as well as the relative error between them. The spread time from the first battery to the second battery, that from the second battery to the third battery, and that from the third battery to the fourth battery are denoted as $t_{1-2}, t_{2-3}$, and $t_{3-4}$, respectively. As shown in Figure 7 and Table 6 , the thermal runaway simulation time simulated by our model agrees well with experimental results, and the error was controlled within $10 \%$.

Table 6. Thermal runaway spread time

\begin{tabular}{cccc}
\hline Time & $t_{1-2}$ & $t_{2-3}$ & $t_{3-4}$ \\
\hline Experimental value & 95 & 112 & 118 \\
Simulation value & 100 & 110 & 108 \\
Relative error & $6.5 \%$ & $-2.9 \%$ & $-8.61 \%$ \\
\hline
\end{tabular}

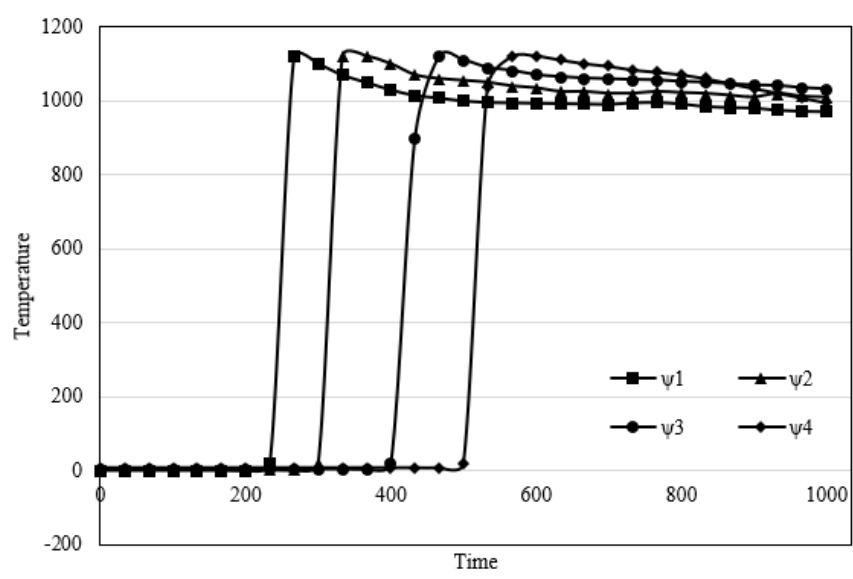

Figure 7. Experimental curve of internal temperature

\section{CONCLUSIONS}

This paper extensively explores the features and spread mechanism of thermal runaway for electric car batteries. Specifically, the acquisition of TH parameters of the batteries was detailed, and the heat parameters in the ARC chamber were recognized, as well as the thermal parameters of battery debris. After that, the analysis was divided into four stages according to the sequence of secondary electrochemical reactions, the thermal runaway features of the batteries were analyzed, and the internal heat yield of the batteries was calculated under each stage of thermal runaway. Next, the thermal runaway spread of batteries was modeled. Through experiments, the authors compared the temperature changes at the battery detection point under different heat source temperatures, coefficients of heat release, and charging speeds, and drew the relevant conclusions. In addition, the mean experimental results were compared with the mean simulation results, revealing the accuracy of the proposed analysis model for thermal runaway and spread of batteries. Finally, the proposed model was applied to compute the thermal runaway 
spread of battery pack, and the effectiveness and precision of our model were further confirmed by comparing measured spread time and internal temperature with the simulation values.

\section{ACKNOWLEDGMENT}

Xingtai Science and Technology Planning Project: "Research and Development of vehicle Controller for Pure Electric City Sweeper”. Project Number: $2019 Z$ C019.

\section{REFERENCES}

[1] Zhang, X., Liu, C., Rao, Z. (2018). Experimental investigation on thermal management performance of electric vehicle power battery using composite phase change material. Journal of Cleaner Production, 201: 916-924. https://doi.org/10.1016/j.jclepro.2018.08.076

[2] Wang, T., Wu, X., Xu, S., Hofmann, H., Du, J., Li, J., Song, Z. (2018). Performance of plug-in hybrid electric vehicle under low temperature condition and economy analysis of battery pre-heating. Journal of Power Sources, 401: 245-254.

https://doi.org/10.1016/j.jpowsour.2018.08.093

[3] Han, B., Liu, F., Li, M., Guo, J., Xu, Y. (2021). Research on electric vehicle thermal management system with coupled temperature regulation between crew cabin and power battery pack. Proceedings of the Institution of Mechanical Engineers, Part D: Journal of Automobile Engineering, 0954407021996581 https://doi.org/10.1177/0954407021996581

[4] Song, D.F., Yang, L.L., Zeng, X.H., Wang, X.Q., Liang, W.Z., Yang, N.N. (2021). Battery life optimization of hybrid electric vehicle based on driving cycle construction. Jilin Daxue Xuebao (Gongxueban)/Journal of Jilin University (Engineering and Technology Edition), 51(3): 781-791.

[5] Liu, Y., Zhang, J. (2021). Electric vehicle battery thermal and cabin climate management based on model predictive control. Journal of Mechanical Design, 143(3): 031705. https://doi.org/10.1115/1.4048816

[6] Xu, M., Wu, T., Tan, Z. (2021). Electric vehicle fleet size for carsharing services considering on-demand charging strategy and battery degradation. Transportation Research Part C: Emerging Technologies, 127: 103146. https://doi.org/10.1016/j.trc.2021.103146

[7] Chen, M., Li, J. (2021). Experimental study on heating performance of pure electric vehicle power battery under low temperature environment. International Journal of Heat and Mass Transfer, 172: 121191. https://doi.org/10.1016/j.ijheatmasstransfer.2021.121191

[8] Ananthraj, C.R., Ghosh, A. (2021). Battery management system in electric vehicle. In 2021 4th Biennial International Conference on Nascent Technologies in Engineering (ICNTE), https://doi.org/10.1109/ICNTE51185.2021.9487762

[9] Sun, Y., Wang, Y. (2021). Structural design and thermal performance analysis of hybrid electric vehicle battery pack cooling system. In Journal of Physics: Conference Series, 1941(1): 012078.

[10] Ding, S., Dong, C., Zhao, T., Koh, L., Bai, X., Luo, J. (2020). A meta-learning based multimodal neural network for multistep ahead battery thermal runaway forecasting. IEEE Transactions on Industrial Informatics, 17(7): 4503-4511. https://doi.org/10.1109/TII.2020.3015555

[11] Zhang, Y., Mei, W., Qin, P., Duan, Q., Wang, Q. (2021). Numerical modeling on thermal runaway triggered by local overheating for lithium iron phosphate battery. Applied Thermal Engineering, 192: 116928. https://doi.org/10.1016/j.applthermaleng.2021.116928

[12] Spray, R., Barry, M., Vickery, J. (2019). Understanding downstream risk from lithium-ion battery thermal runaway \& designing for safety. ECS Transactions, 89(1): 65.

[13] Chen, M., Ouyang, D., Weng, J., Liu, J., Wang, J. (2019). Environmental pressure effects on thermal runaway and fire behaviors of lithium-ion battery with different cathodes and state of charge. Process Safety and Environmental Protection, 130: 250-256. https://doi.org/10.1016/j.psep.2019.08.023

[14] Harrison, J., Charles, D., Zenker, J., Frank, E. (2019). Using multi-physics system simulation to predict battery pack thermal performance and risk of thermal runaway during eVTOL aircraft operations. In 2019 AIAA/IEEE Electric Aircraft Technologies Symposium (EATS), 1-13. https://doi.org/10.2514/6.2019-4406

[15] Kshetrimayum, K.S., Yoon, Y.G., Gye, H.R., Lee, C.J. (2019). Preventing heat propagation and thermal runaway in electric vehicle battery modules using integrated PCM and micro-channel plate cooling system. Applied Thermal Engineering, 159: 113797. https://doi.org/10.1016/j.applthermaleng.2019.113797

[16] Yokoshima, T., Mukoyama, D., Maeda, F., Osaka, T., Takazawa, K., Egusa, S., Yamamoto, K. (2018). Direct observation of internal state of thermal runaway in lithium ion battery during nail-penetration test. Journal of Power Sources, 393: 67-74. https://doi.org/10.1016/j.jpowsour.2018.04.092

[17] Le, A.V., Wang, M., Noelle, D.J., Shi, Y., Qiao, Y. (2018). Mitigating thermal runaway of lithium-ion battery by using thermally sensitive polymer blend as cathode binder. Journal of Applied Polymer Science, 135(4): 45737.

[18] Recoskie, S., MacNeil, D.D., Kodra, O., Torlone, G., Lalime, G., Hendershot, K. (2017). Thermal runaway initiation and propagation within $\mathrm{xEV}$ battery packs. Application and Market and Chemistry and Materials for Lead-Based Batteries.

[19] Sheikh, M., Elmarakbi, A., Elkady, M. (2017). Thermal runaway detection of cylindrical 18650 lithium-ion battery under quasi-static loading conditions. Journal of Power Sources, 370: 61-70. https://doi.org/10.1016/j.jpowsour.2017.10.013

[20] Hong, J., Wang, Z., Liu, P. (2017). Big-data-based thermal runaway prognosis of battery systems for electric vehicles. $\quad$ Energies, $10(7)$ : 919. https://doi.org/10.3390/en10070919

[21] Zhang, W., Liang, Z., Wu, W., Ling, G., Ma, R. (2021). Design and optimization of a hybrid battery thermal management system for electric vehicle based on surrogate model. International Journal of Heat and Mass Transfer, 174: 121318. https://doi.org/10.1016/j.ijheatmasstransfer.2021.12131 8

[22] Afzal, A., Samee, A.M., Razak, R.A., Ramis, M.K. 
(2021). Thermal management of modern electric vehicle battery systems (MEVBS). Journal of Thermal Analysis and Calorimetry, 144(4): 1271-1285. https://doi.org/10.1007/s10973-020-09606-x

[23] Burd, J.T.J., Moore, E.A., Ezzat, H., Kirchain, R., Roth, R. (2021). Improvements in electric vehicle battery technology influence vehicle lightweighting and material substitution decisions. Applied Energy, 283: 116269. https://doi.org/10.1016/j.apenergy.2020.116269

[24] Wang, Z., He, T., Bian, H., Jiang, F., Yang, Y. (2021). Characteristics of and factors influencing thermal runaway propagation in lithium-ion battery packs.
Journal of Energy Storage, 41: 102956. https://doi.org/10.1016/j.est.2021.102956

[25] Jiang, L., Deng, Z., Tang, X., Hu, L., Lin, X., Hu, X. (2021). Data-Driven Fault Diagnosis and Thermal Runaway Warning for Battery Packs Using Real-World Vehicle Data. Energy, 121266.

[26] Li, Y., Qi, F., Guo, H., Guo, Z., Xu, G., Liu, J. (2019). Numerical investigation of thermal runaway propagation in a Li-ion battery module using the heat pipe cooling system. Numerical Heat Transfer, Part A: Applications, 75(3):

https://doi.org/10.1080/10407782.2019.1580956 